\title{
HTS Step-edge Josephson Junction Terahertz Harmonic Mixer
}

Jia $\mathrm{Du}^{1}$, Andrew R. Weily ${ }^{2}$, Xiang Gao ${ }^{1}$, Ting Zhang ${ }^{2}$, Cathy P. Foley ${ }^{1}$, Yingjie Jay Guo ${ }^{3}$

${ }^{1}$ CSIRO Manufacturing, PO Box 218, Lindfield, NSW 2070, Australia;

${ }^{2}$ CSIRO Data 61, Marsfield, NSW 1710, Australia;

${ }^{3}$ Global Big Data Technologies Centre, University of Technology, Sydney, Ultimo, NSW 2007, Australia

\begin{abstract}
A high-temperature superconducting (HTS) terahertz $(\mathrm{THz})$ frequency down-converter or mixer based on a thin-film ring-slot antenna coupled $\mathrm{YBa}_{2} \mathrm{Cu}_{3} \mathrm{O}_{7-x}(\mathrm{YBCO}) / \mathrm{MgO}$ step-edge Josephson junction is reported. The frequency down-conversion was achieved using higher order harmonics of an applied lower frequency $(19-40 \mathrm{GHz})$ local oscillator signal in the Josephson junction mixing with a $\mathrm{THz}$ signal of over $600 \mathrm{GHz}$, producing a 1-3 GHz intermediate frequency signal. Up to 31st order of harmonic mixing was obtained and the mixer operated stably at temperatures up to $77 \mathrm{~K}$. The design details of the antenna, HTS Josephson mixer, the matching and isolation circuits, and the DC and RF performance evaluation are described in this paper.
\end{abstract}

\section{Introduction}

The unprecedented growth in the development of wireless communication systems has pushed operating frequencies towards the millimetre and lower terahertz $(\mathrm{THz})$ frequency range. The large amount of bandwidth available at $\mathrm{THz}$ frequencies will enable fast transmission of huge amounts of data as needed for new emerging applications. Research on $\mathrm{THz}$ communications has received increased attention in recent years [1]. New developments to improve the performance of sources and detectors, circuits, antenna technology, and system architecture are required to realize ultrafast $\mathrm{THz}$ data transmission. Due to extensive transmission loss in the atmosphere of millimetre and $\mathrm{THz}$ waves, ultrahigh-sensitive heterodyne receiver front-ends or mixers are needed to compensate for the propagation loss. 
High-temperature superconducting (HTS) Josephson junctions are promising candidates for $\mathrm{THz}$ band detectors and mixers due to their special properties, such as low noise, low power consumption, broadband and high frequency operation (well into $\mathrm{THz}$ band) as a result of the highenergy band gap. Compared to the low-temperature superconducting (LTS) devices, HTS devices offer an obvious practical advantage of operating at higher temperatures so that cheaper and simpler cryogenic instruments (for example, miniature size portable cryocoolers) can be used. HTS $\mathrm{THz}$ direct detectors based on bicrystal $[2,3]$ and step-edge $[4,5]$ grain-boundary junctions have been developed in recent years. These HTS detectors are, however, not suitable for use in communication systems as they only measure the amplitude of $\mathrm{THz}$ signal power not the phase information that is required for telecommunication applications. Recently, HTS Josephson heterodyne frequency down-converters or mixers based on CSIRO step-edge junction technology were demonstrated in the microwave frequency range up to $30 \mathrm{GHz}[6,7]$.

There have been very limited reports of successful demonstration of HTS Josephson junction THz mixers [8 -10] due to the challenges of producing reliable and high quality HTS Josephson junctions and inaccessible instruments and components at $\mathrm{THz}$ frequency bands. The reported HTS THz mixers in the literature mostly operate at temperatures significantly lower than 77K (for example, 10 and $20 \mathrm{~K}[8,9]$ and $50 \mathrm{~K}[10])$ and at frequencies lower than reported in this paper. For heterodyne mixing in the submillimeter and $\mathrm{THz}$ wave regions, it is difficult to obtain a tunable high frequency local oscillator (LO) for fundamental mixing. Consequently, Josephson junction harmonic mixing would be a particularly useful technique. Josephson junction harmonic mixers have been mostly observed in LTS SIS (superconductor-insulator-superconductor) junctions at microwave and millimeter frequencies [11, 12]. Chen et al [13] observed high order harmonic mixing well into $\mathrm{THz}$ frequency range in HTS bicrystal junctions, but no details of mixer performance were given. Harmonic mixing

In this paper, we report our first demonstration of a HTS step-edge junction THz heterodyne mixer using high-order harmonic mixing. The advantage of such a harmonic mixer is to remove the need of using a second expensive $\mathrm{THz}$ signal source as the LO pumping source thus simplifying the system architecture and reducing the system cost and size. A thin-film antenna and microwave matching circuit were designed to couple the THz signal and LO signal into the Josephson junction mixer and extract the down-converted IF signal.

\section{Design of Antenna, Matching and Isolation Circuits for the Josephson Harmonic Mixer}

The schematic layout of the antenna-coupled HTS Josephson junction mixer and matching circuit is shown in Figure 1. The aims of the mixer and bias-tee design are to create a low-loss path from the 
Josephson junction mixer to the LO and IF ports, while maintaining high isolation between the RF, LO, IF and DC ports. The mixer is fabricated on a $0.5 \mathrm{~mm}$ thick MgO substrate $\left(\varepsilon_{\mathrm{r}}=9.65, \tan \delta=\right.$ $0.0005)$, which then couples to a $3 \mathrm{~mm}$ diameter high resistivity silicon hemispherical lens $\left(\varepsilon_{\mathrm{r}}=11.9\right.$, $\tan \delta=0.00025)$. The step-edge Josephson junction is placed across the circular slot of a thin-film ring-slot antenna as shown in the enlarged inset in Figure 1. The ring-slot antenna has a truncated circular ground plane and a coplanar waveguide (CPW) feed network that attaches to a CPW lowpass filter (LPF) with a $-3 \mathrm{~dB}$ cut-off frequency of approximately $250 \mathrm{GHz}$. This ring-slot/CPW combination is similar to that used previously for amplitude detection [4], but has been adapted here for use as a harmonic mixer and its parameters optimized to give peak realized gain around 620 to $640 \mathrm{GHz}$. The LPF is connected to a tapered CPW line for efficient coupling to the bias-tee circuit, such that the width of the CPW centre conductor matches the width of the microstrip line with a characteristic impedance of $50 \Omega$. The tapered CPW line also acts as a LPF, with a -3dB cut-off frequency of approximately $100 \mathrm{GHz}$, and serves to filter the $\mathrm{THz}$ signal received by the ring-slot antenna from the LO and IF ports.

The bias-tee was designed to provide isolation between the DC bias current applied to the HTS junction and the LO and IF signals of the mixer circuit, and to be wideband to enable flexibility in the choice of the LO frequency that drives the mixer. It was implemented on a $254 \mu \mathrm{m}$ thick polished Alumina substrate $\left(\varepsilon_{\mathrm{r}}=9.9, \tan \delta=0.0002\right)$ with a $3 \mu \mathrm{m}$ TiW/Au metallization. Both the bias circuit and ring-slot antenna were designed using the software CST Microwave Studio. To the left of the bias-tee is a diplexer circuit, that consists of a high-pass filter (HPF) and a LPF to separate the LO and IF signals. This circuit is located external to the cryocooler, it has a LPF passband from DC to $17 \mathrm{GHz}$, a HPF passband from $\sim 19$ to $40 \mathrm{GHz}$, and a typical isolation of $50 \mathrm{~dB}$.

In the mixer prototype, two ring-slot antennas are included in a single $10 \mathrm{~mm} \times 10 \mathrm{~mm}$ substrate and the schematic of the mixer prototype is shown in Figure 2. A single hemispherical lens of $3 \mathrm{~mm}$ diameter is placed on the back of the substrate. The centre-to-centre distance between the two ringslot antennas is $300 \mu \mathrm{m}$, which makes the offset from the ring-slot to centre of the lens $150 \mu \mathrm{m}$. The port labels are used for analysis using CST Microwave Studio. Ports 1 and 3 correspond to the location of the Josephson junctions, and the normal resistance $R_{\mathrm{n}}$ of the junction has been estimated to be between $2 \Omega$ and $4 \Omega$. Ports 2 and 4 correspond to the CPW transmission line ports, which connect to bias-tees as described in Figure 1. Computed realized gain of the lens/ring-slot antenna combination as a function of frequency for two different values of the junction resistance $R_{\mathrm{n}}$ is presented in Figure 3, where realized gain includes both ohmic and mismatch losses due to the low normal resistance of the junction, and Port 1 is excited. It can be seen that higher $R_{\mathrm{n}}$ value results in 
better antenna gain due to better impedance matching. In this experiment, an $R_{\mathrm{n}}$ value of $4 \Omega$ was measured. A peak realized gain of $18.2 \mathrm{dBi}$ is computed at $640 \mathrm{GHz}$, and the realized gain is greater than $15 \mathrm{dBi}$ from 590 to $670 \mathrm{GHz}$. Radiation patterns for the E-plane have also been computed for the lens/ring-slot antenna and are depicted in Figure 4 . There is a $15^{\circ}$ beam squint in the E-plane due to the 150 um offset of the ring-slot antenna from the centre of the lens. Computed half power beamwidth (HPBW) is $11^{\circ}$ in both the E-plane and H-plane at $620 \mathrm{GHz}$. To show the coupling efficiency, the S-parameters of the dual ring-slot feed have also been computed and are shown in Figure 5. Coupling $\left(\mathrm{S}_{21}\right)$ from the Josephson junction at P1 to P2 shows the loss in the LO and IF signal chains and is $5 \pm 1 \mathrm{~dB}$ from 0 to $30 \mathrm{GHz}$. Coupling from $\mathrm{P} 1$ to the ports of the adjacent ring-slot antenna ( $\mathrm{P3}$ and $\mathrm{P} 4)$ is also important to show there is sufficient isolation between the two antennas. Figure 5 shows that the RF isolation $\left(S_{31}\right)$ between the Josephson junction ports is greater than $25 \mathrm{~dB}$ at $620 \mathrm{GHz}$, and the LO/IF isolation $\left(\mathrm{S}_{41}\right)$ of Port 4 from the RF signal at Port 1 is better than $50 \mathrm{~dB}$ from 0 to $30 \mathrm{GHz}$. The computed reflection coefficient, $S_{11}$, at the ring-slot $(P 1)$ is $-2.8 \mathrm{~dB}$ at $620 \mathrm{GHz}$; this value is low due to the relatively small normal resistance of the Josephson junction. Improvement to the matching can be achieved by further increasing the junction normal resistance, a subject of the on-going HTS junction parameter optimisation.

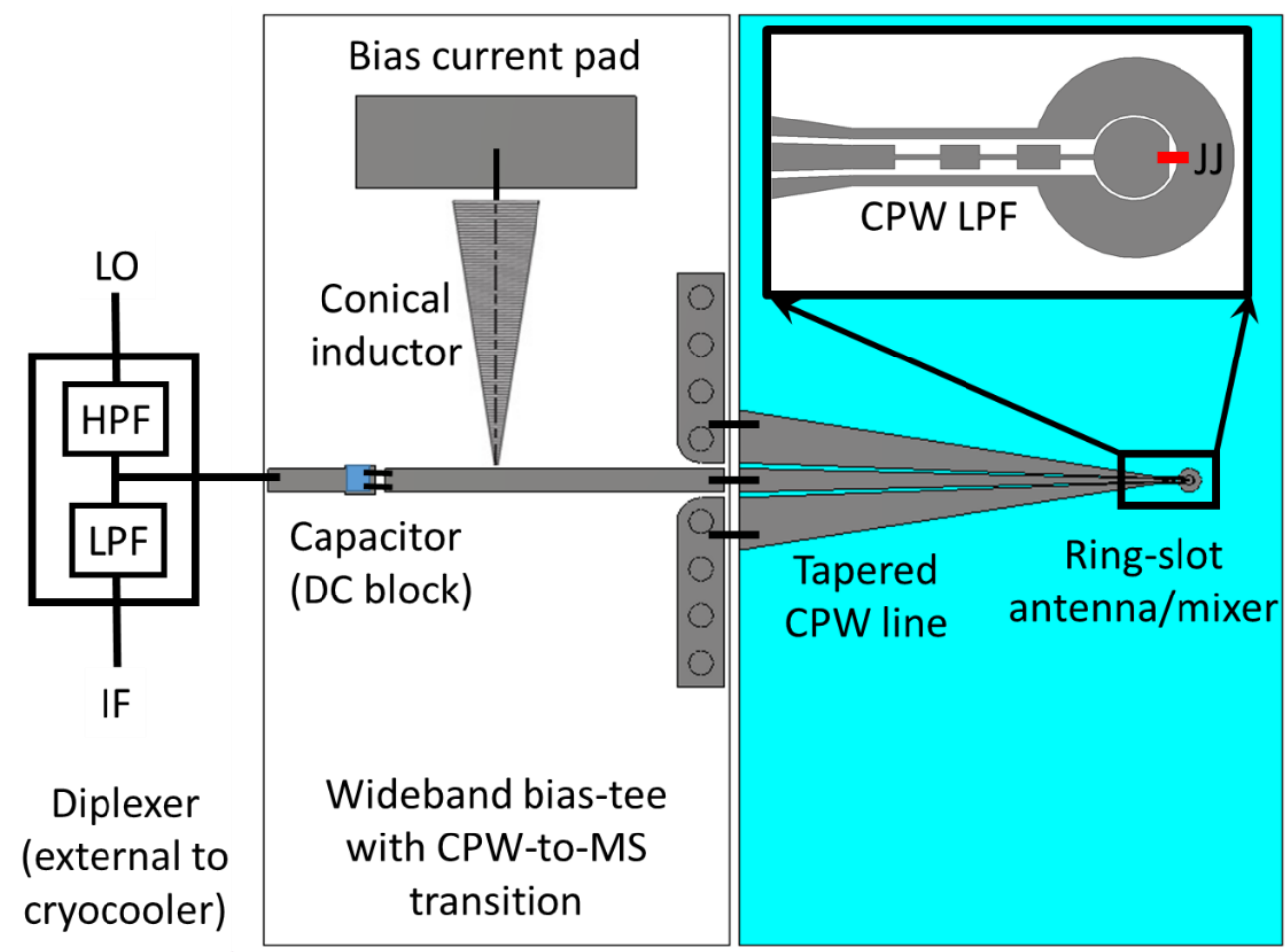

Figure 1. Schematic diagram of THz harmonic mixer. Inset shows an enlarged view of the THz ringslot antenna and the location of the Josephson junction (JJ). 


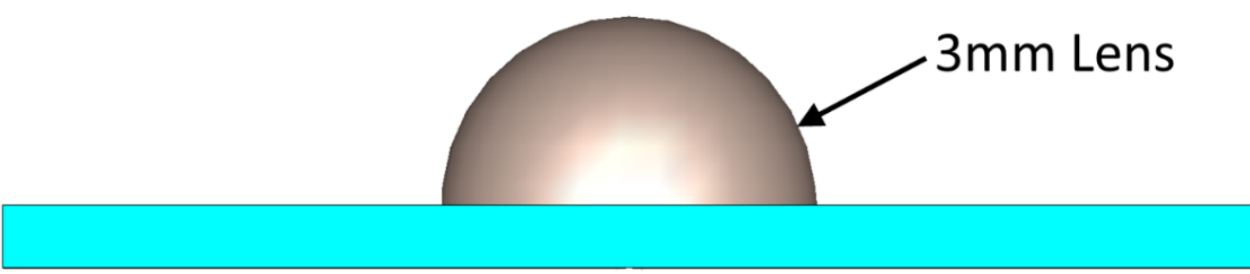

Side View

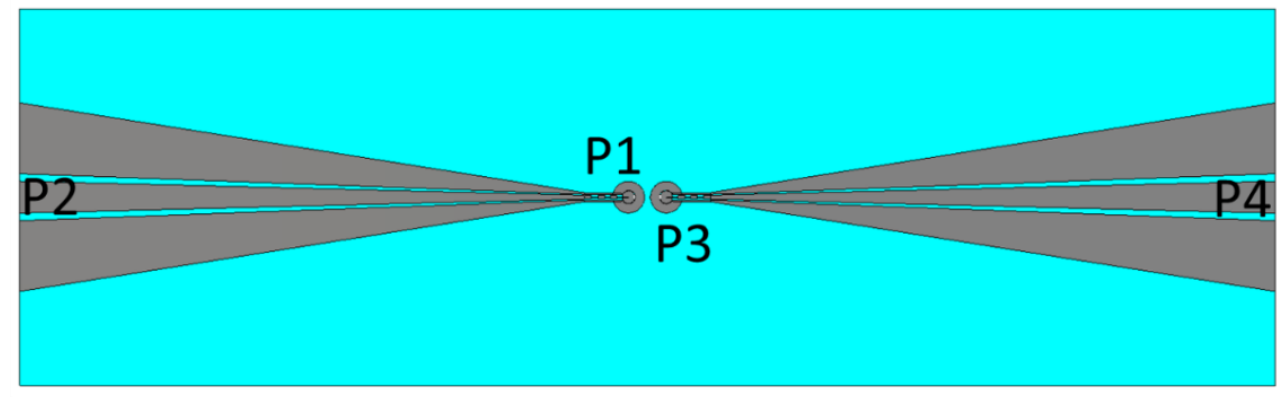

Bottom View

Figure 2. Side view and bottom view of two $\mathrm{THz}$ ring-slot antennas coupled to a single $3 \mathrm{~mm}$ hemispherical lens, including port number labels.

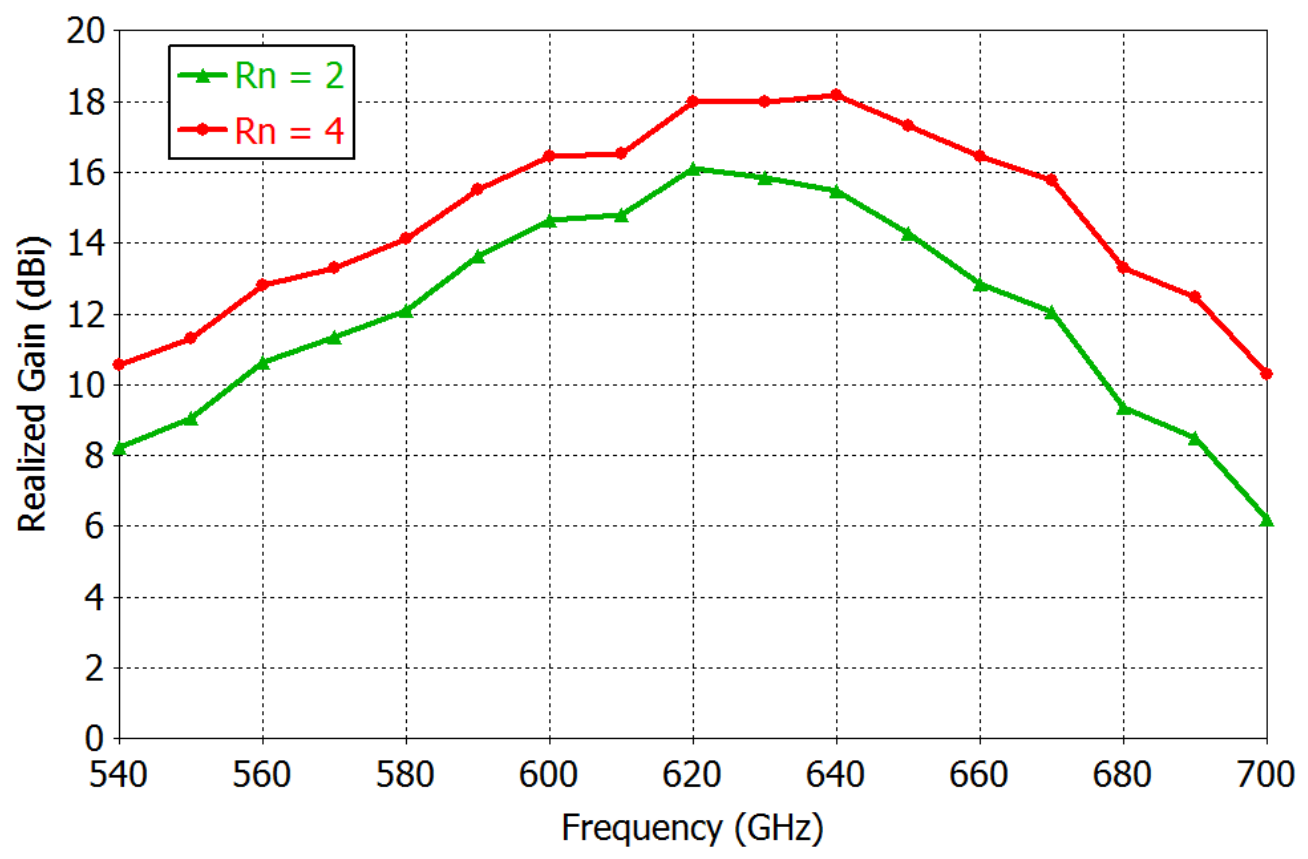

Figure 3. Computed realized gain of the THz ring-slot antenna with the lens for two difference $R_{\mathrm{n}}$ values of the HTS junction. 


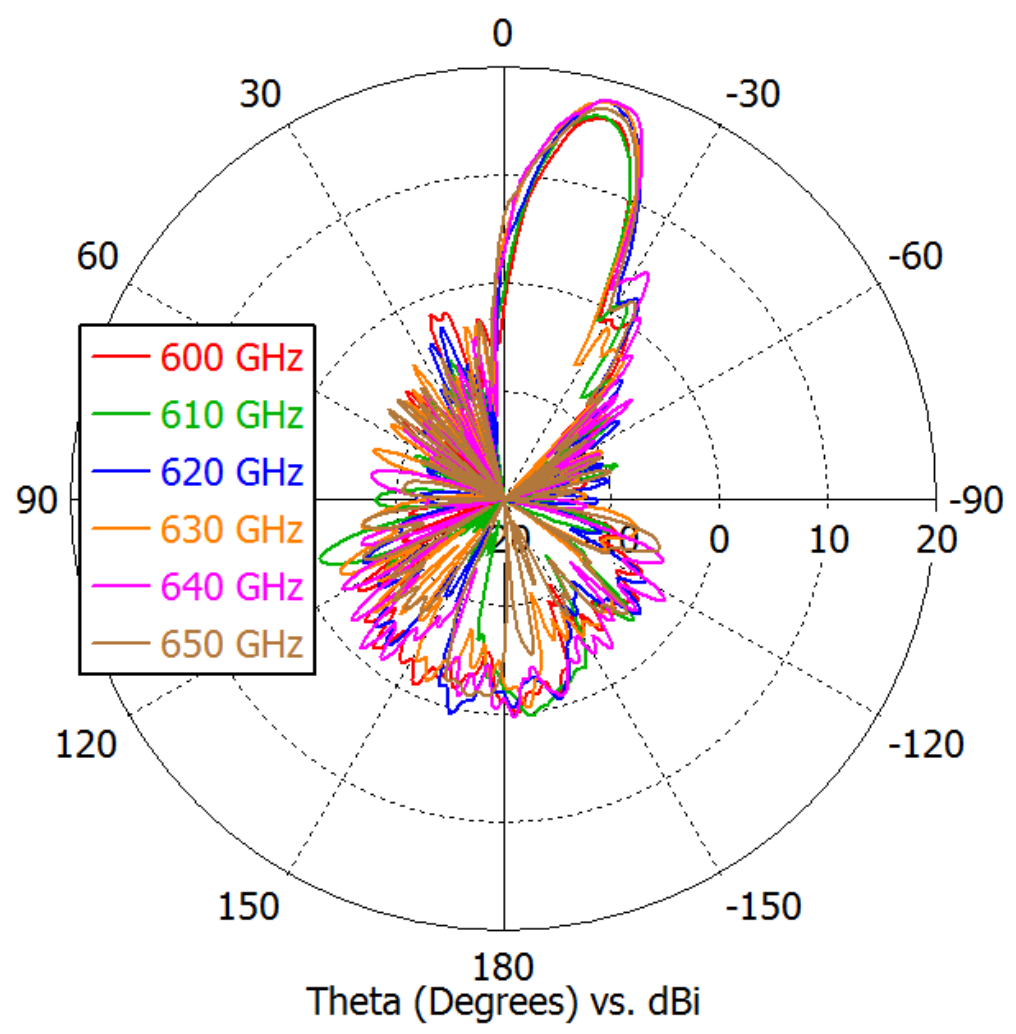

Figure 4. Computed E-plane radiation patterns of the $\mathrm{THz}$ ring-slot antenna from 600 to $650 \mathrm{GHz}$.

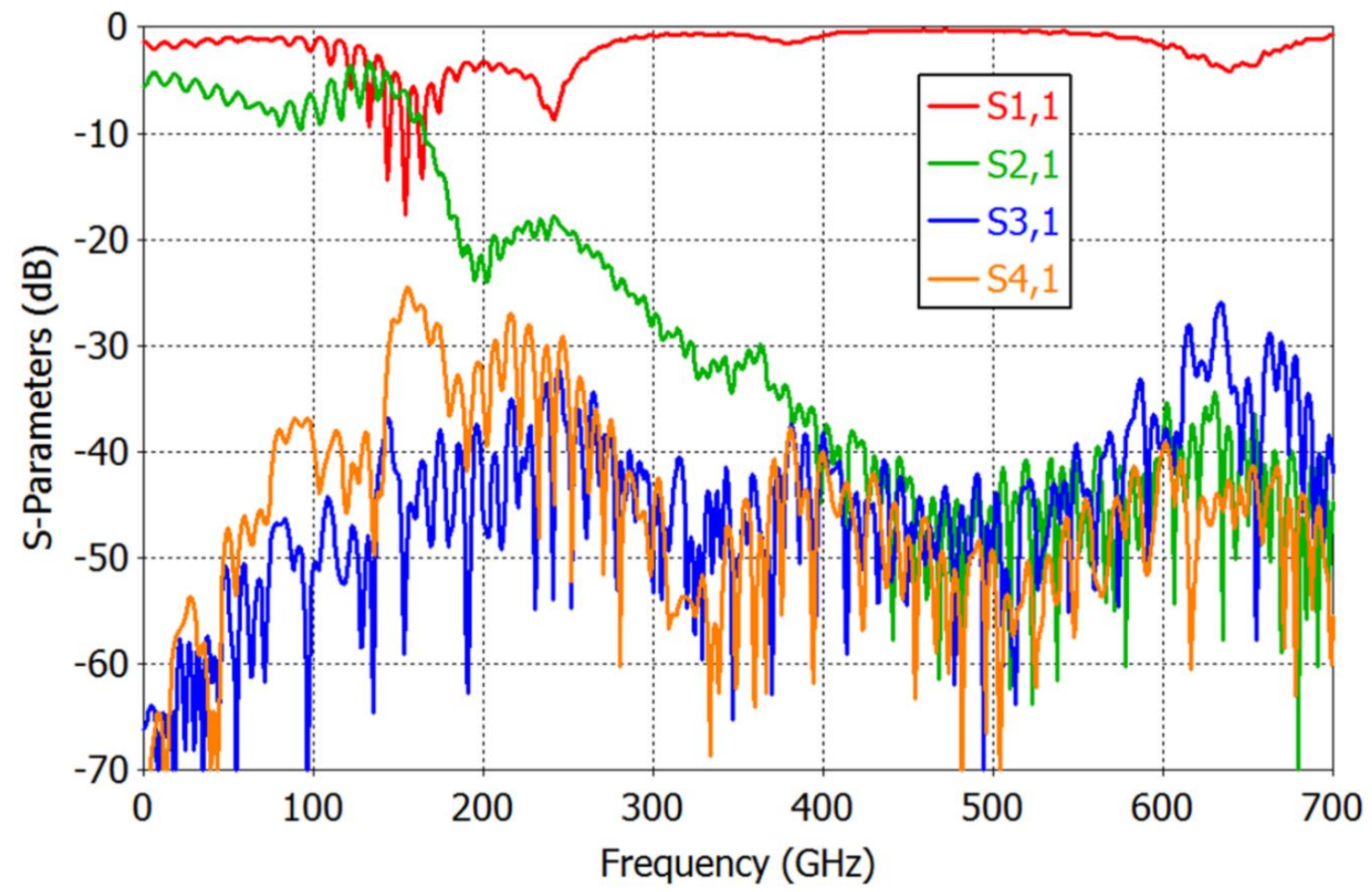

Figure 5. Computed S-parameters of two THz ring-slot antennas coupled to a single $3 \mathrm{~mm}$ lens. 


\section{Experimental Details}

The device was fabricated using the established step-edge $\mathrm{YBa}_{2} \mathrm{Cu}_{3} \mathrm{O}_{7-x}(\mathrm{YBCO})$ junction technology developed at CSIRO $[14,15]$. The $10 \mathrm{~mm} \times 10 \mathrm{~mm} \times 0.5 \mathrm{~mm} \mathrm{MgO}$ substrate was first patterned and etched using a standard photolithography and Ar-ion beam etching techniques to create a step-edge pattern with $\sim 400 \mathrm{~nm}$ height and $\sim 35^{\circ}$ angle. A $200 \mathrm{~nm}$ thick YBCO film with a $50 \mathrm{~nm}$ in-situ Au film on top was deposited on the processed $\mathrm{MgO}$ substrate by Ceraco $\mathrm{GmbH}$. The YBCO film was then patterned and etched to form the step-edge junctions and the DC bias lines. The in-situ Au was removed from the junction area but remained on the DC lines and contact pads for achieving lower contact resistance and bondable pads [16]. Additional layer of $\sim 300 \mathrm{~nm}$ Au thin film was deposited using dc sputtering technique and patterned to form the Au thin-film antenna.

Figure 6 shows the micrographs of the fabricated chip ((a) and (b)) and the photographs of the packaged mixer module ((c) and (d)). The ring slot antennas with the tapered CPW lines are made from the Au thin-film and the Josephson junctions are the $2 \mu \mathrm{m}$ wide narrow YBCO micro strips across the step patterns located in the ring slots as shown in the enlarged view in (b). Two mixer devices were made on a single $10 \times 10 \mathrm{~mm} \mathrm{MgO}$ substrate with the in-house designed bias-tee on both sides to obtain two mixer modules in one package. The fabricated YBCO/MgO substrate with two PCB bias tee circuits were mounted into a specially designed Au-coated copper housing. The series inductors and shunt capacitors were mounted on the bias tee PCBs ((c)) which are used to block the DC signals from the LO and IF ports and to block the RF, LO and IF signals from the DC bias port. DC and RF wire connections were made using ultrasonic Au ribbon bonding. The top eight colored wires in Figure (c) are the DC bias lines connected via surface mount capacitors and resistors. As shown in Figure (d), a $3 \mathrm{~mm}$ diameter high resistivity silicon hyper-hemispherical lens was bonded to the reverse side of the substrate for coupling the $\mathrm{THz}$ signal. The combination of the MgO substrate and hemispherical silicon lens forms a lens which ensures a high antenna directivity and efficient coupling between free-space and the antennas.

The packaged THz HTS mixer was cooled on a commercial 2-stage pulse-tube cryocooler (PTC). In this experiment, the DC and RF mixing properties were characterized at operating temperatures from 40 to $77 \mathrm{~K}$, a temperature range that is potentially attainable with a single-stage mini cryocooler thus demonstrating the full potential of a HTS THz mixer for practical applications. A compact commercial solid-state THz source (VDI 625 GHz Amplifier Multiplier Chain) was used to generate $\mathrm{THz}$ radiation between 590 and $650 \mathrm{GHz}$. The THz signal, after passing through a pair of collimating and focusing mirrors, was focused onto the HTS mixer module via the Si lens through the cryocooler window. The LO pumping signal was supplied using an Agilent Technologies E8257D signal generator 
for frequencies up to $20 \mathrm{GHz}$ or an Anritsu $68087 \mathrm{C}$ signal generator for frequencies up to $40 \mathrm{GHz}$. For harmonic mixing, the LO and IF signals share the same port of the mixer module and the IF signal was isolated by using a microwave diplexer connected outside of the cryocooler. The mixer IF output was amplified using a room temperature low-noise-amplifier (LNA) and displayed on an Agilent E4407B spectrum analyzer. A battery-powered DC current source was used to bias the Josephson mixer.
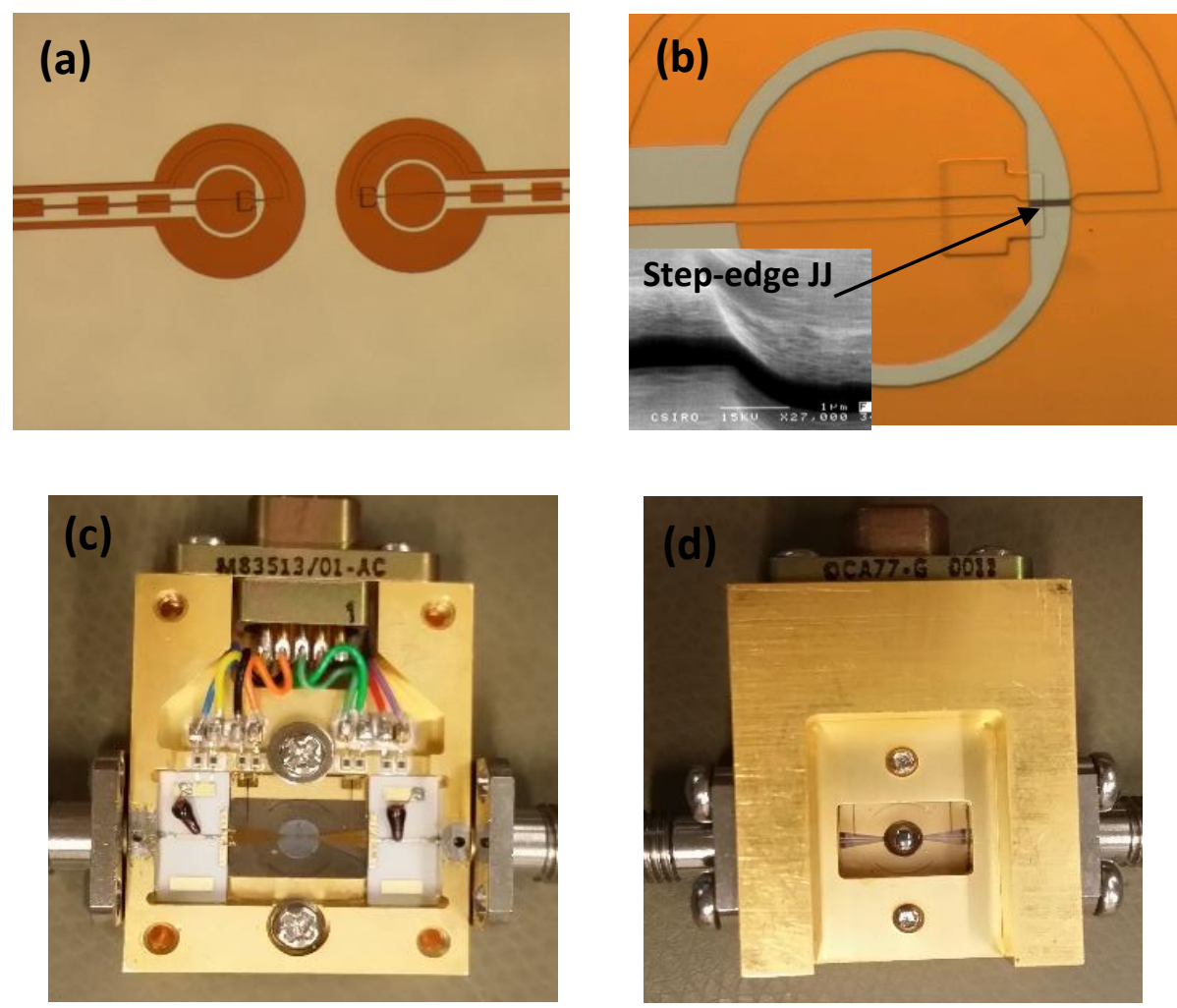

Figure $6(\mathrm{a})$ and (b) are photographs of the fabricated device chip containing two antenna-coupled HTS Josephson mixers; (b) shows a $2 \mu \mathrm{m}$ wide YBCO micro-strip line (black colour) across a step-edge on the $\mathrm{MgO}$ substrate inside the slot forming a step-edge junction which connects the inner and outer conductors of the antenna. The inset in (b) is a scanning electron micrograph (SEM) graph of the step-edge junction. (c) and (d) are photographs of the packaged module showing on the circuit side (c) and the backside (d) where a $3 \mathrm{~mm}$ Si lens is attached onto the back of MgO substrate for coupling the $\mathrm{THz}$ signal onto the antenna and junction.

\section{Results and Discussion}

Figure 7 shows the DC current-voltage characteristics (IVCs) of the step-edge junction at different operating temperatures from $40 \mathrm{~K}$ to $77 \mathrm{~K}$. The junction demonstrated a resistively-shunted-junction behaviour with a normal resistance $R_{\mathrm{n}} \approx 4 \Omega$. The junction critical current, $I_{c}$, increases with 
decreasing temperature, from $25 \mu \mathrm{A}$ at $77 \mathrm{~K}$ to $500 \mu \mathrm{A}$ at $40 \mathrm{~K}$. The junction characteristic voltage, $V_{\mathrm{c}}$ $\equiv I_{c} R_{\mathrm{n}}$, versus the temperature is plotted in the inset of the figure. The $I_{c} R_{\mathrm{n}}$ value increases from 100 $\mu \mathrm{V}$ at $77 \mathrm{~K}$ to $2 \mathrm{mV}$ at $40 \mathrm{~K}$, which corresponds to a junction characteristic frequency, $f_{\mathrm{c}} \equiv I_{\mathrm{c}} R_{\mathrm{n}} / \Phi_{0}$ (where $\Phi_{0}$ is the magnetic flux quantum, $1 / \Phi_{0} \equiv 2 \mathrm{e} / \mathrm{h}=0.4836 \mathrm{GHz} / \mu \mathrm{V}$ ), of $\sim 50 \mathrm{GHz}$ at $77 \mathrm{~K}$ to $\sim 1$ $\mathrm{THz}$ at $40 \mathrm{~K}$. The characteristic frequency value of the junction shows it is well suited as a $\mathrm{THz}$ frequency down-converter or mixer. By changing operating temperature, the junction parameters can be adjusted to enable RF detection and mixing across a very wide frequency range. This is an additional advantage of using a temperature-adjustable cryocooler instead of liquid nitrogen, though we can tune our fabrication process to optimise the junction characteristics for use at a given fixed temperature.

As shown in Figure 7, the dynamic resistance, $R_{D}=\mathrm{d} V / \mathrm{d} l$, increases with decreasing the temperature which should result in an increase of the mixer conversion efficiency $(\eta)$ as shown by the equation below [17].

$$
\eta=P_{\mathrm{IF}} / P_{\mathrm{RF}}=\frac{C_{I F} R_{D}}{8}\left(\frac{\partial\left(I_{C}\right)}{\partial\left(\mathrm{P}_{\mathrm{LO}}\right)^{1 / 2}}\right)^{2}
$$

where $C_{\text {IF }}$ is the output coupling efficiency $(\leq 1), P_{\text {LO }}$ is the available power from the LO source, $I_{\mathrm{c}}$ is the junction critical current under the operating conditions (suppressed by $P_{\mathrm{LO}}$ ). Eq. (1) shows the conversion efficiency scales with $R_{\mathrm{D}}$ and the differential dependence of $I_{\mathrm{C}}$ on $P_{\mathrm{LO}}$. In this experiment, the mixer operates on high-order of $P_{\mathrm{LO}}$ harmonic mixing and the suppression of $I_{\mathrm{c}}$ by $P_{\mathrm{LO}}$ harmonics $\frac{\partial\left(I_{c}\right)}{\partial\left(\mathrm{P}_{\mathrm{LO}}\right)^{1 / 2}}$ is not a directly measurable parameter. However, the conversion efficiency, $\eta$, may be estimated by measuring $P_{\mathrm{IF}}$ peak of harmonic mixing and the corresponding $\mathrm{THz}$ signal power coupled into the junction using Shapiro steps on the DC IVCs.

Figure 8 shows the IVCs of the Josephson mixer at temperatures from $40 \mathrm{~K}$ to $77 \mathrm{~K}$ under the illumination of a THz signal. Clear Shapiro-steps are induced at the voltages $V_{n}=n \Phi_{0} f_{s}=n \times(1.27$ $m V)(n=\ldots,-2,-1,0,1,2, \ldots)$ and the signal frequency $f_{\mathrm{s}}=614.4 \mathrm{GHz}$. Note that decreasing the operating temperature results in increase of the Shapiro current-step heights (more THz power is coupled into the junction). From the current step-height, $I_{n}$, we can estimate the THz signal power coupled into the Josephson junction, $I_{n} / I_{c}=J_{n}\left(2 e V_{R F} / h f\right)$, where $J_{n}$ is an nth order Bessel function and $V_{\mathrm{RF}}$ is the RF voltage (that is the $\mathrm{THz}$ signal voltage here). This can then be used to estimate the mixer conversion efficiency $\eta=P_{I F} / P_{R F}$. In this experiment, we used a wire-grid polarizer to reduce the $\mathrm{THz}$ signal power in order to operate the $\mathrm{THz}$ mixer in smaller or weak signal range. 


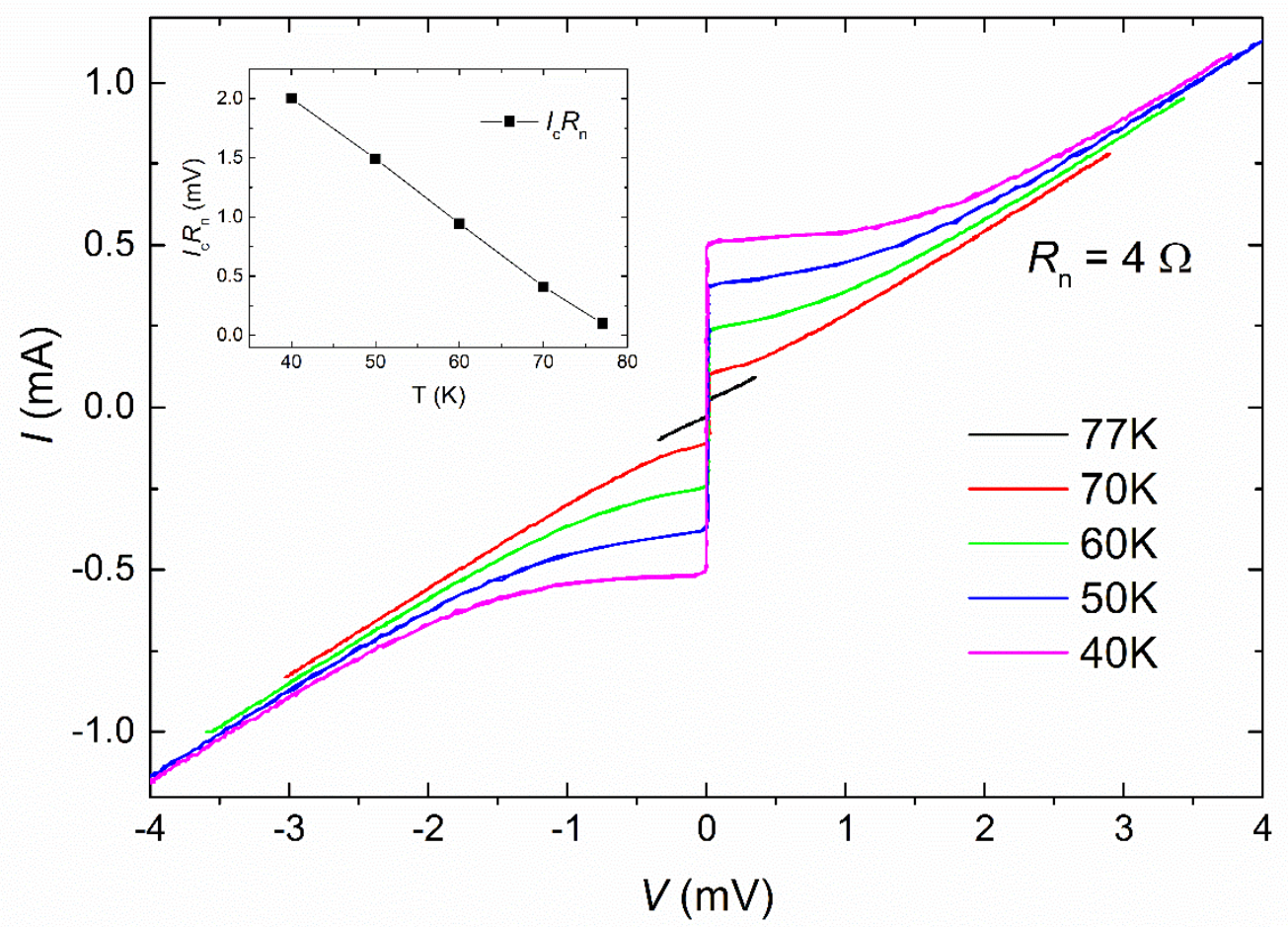

Figure 7. DC IVCs at different temperatures and the inset shows the relationship of the $I_{c} R_{n}$ value against temperature $\mathrm{T}(\mathrm{K})$.

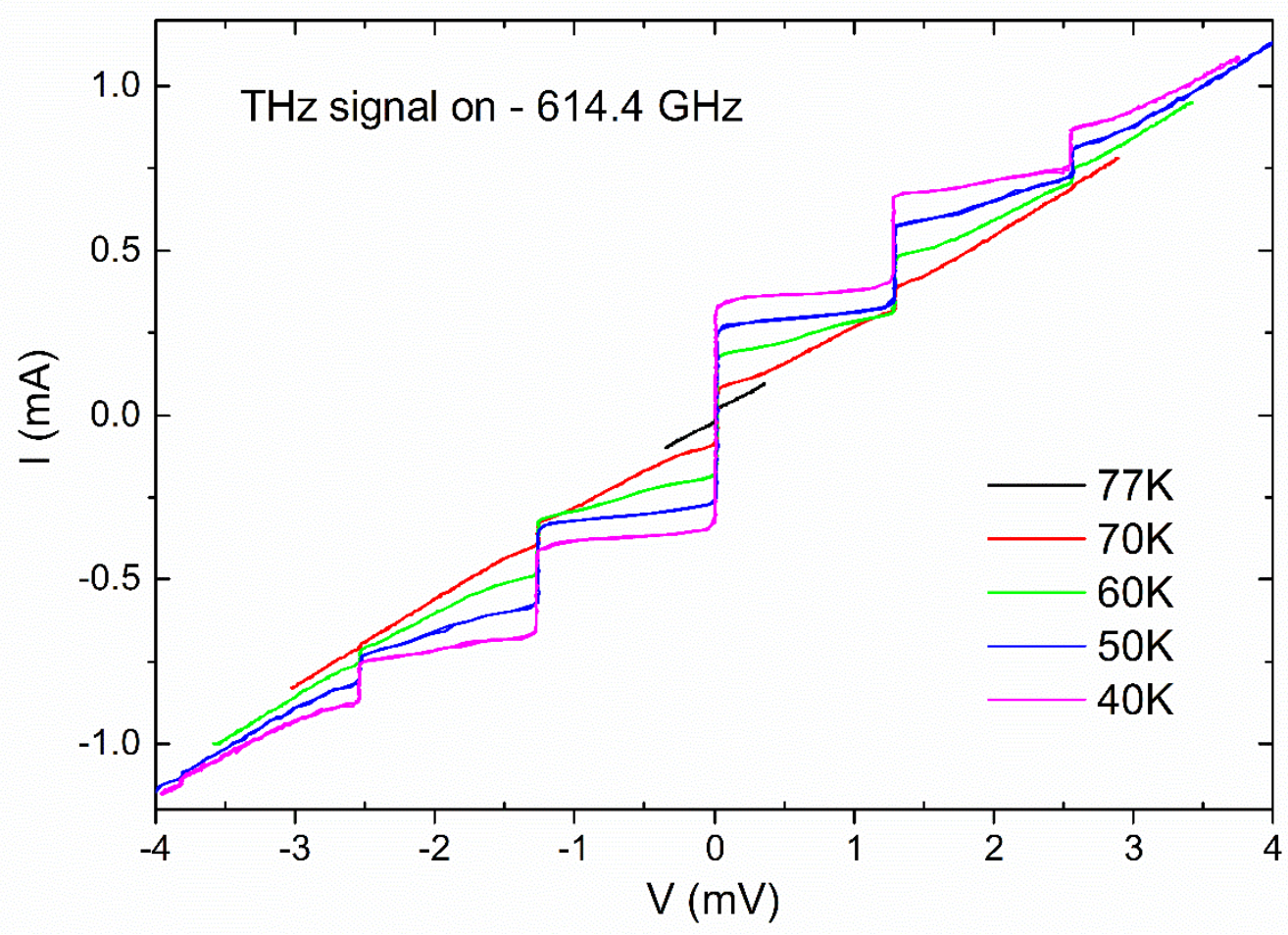

Figure. 8 DC IVCs at different temperatures under illumination of a THz signal. 
Fig. 9 is the IVCs at 40K under applied THz and LO (fundamental) signal powers corresponding to the RF results presented in Figs. 10 and 11 . Junction $I_{c}$ is supressed by both THz signal and LO power. At fixed THz power, the level of LO power affects the IF output and operation range, as shown in the measured mixing optimal bias conditions in Fig. 12. From the 0-order Shapiro step-height induced by the THz signal shown in Fig. 9, we estimated that the THz power coupled into the mixer junction is approximately $47 \mathrm{nW}$ (Note that this is much attenuated THz power using a wire-grid).

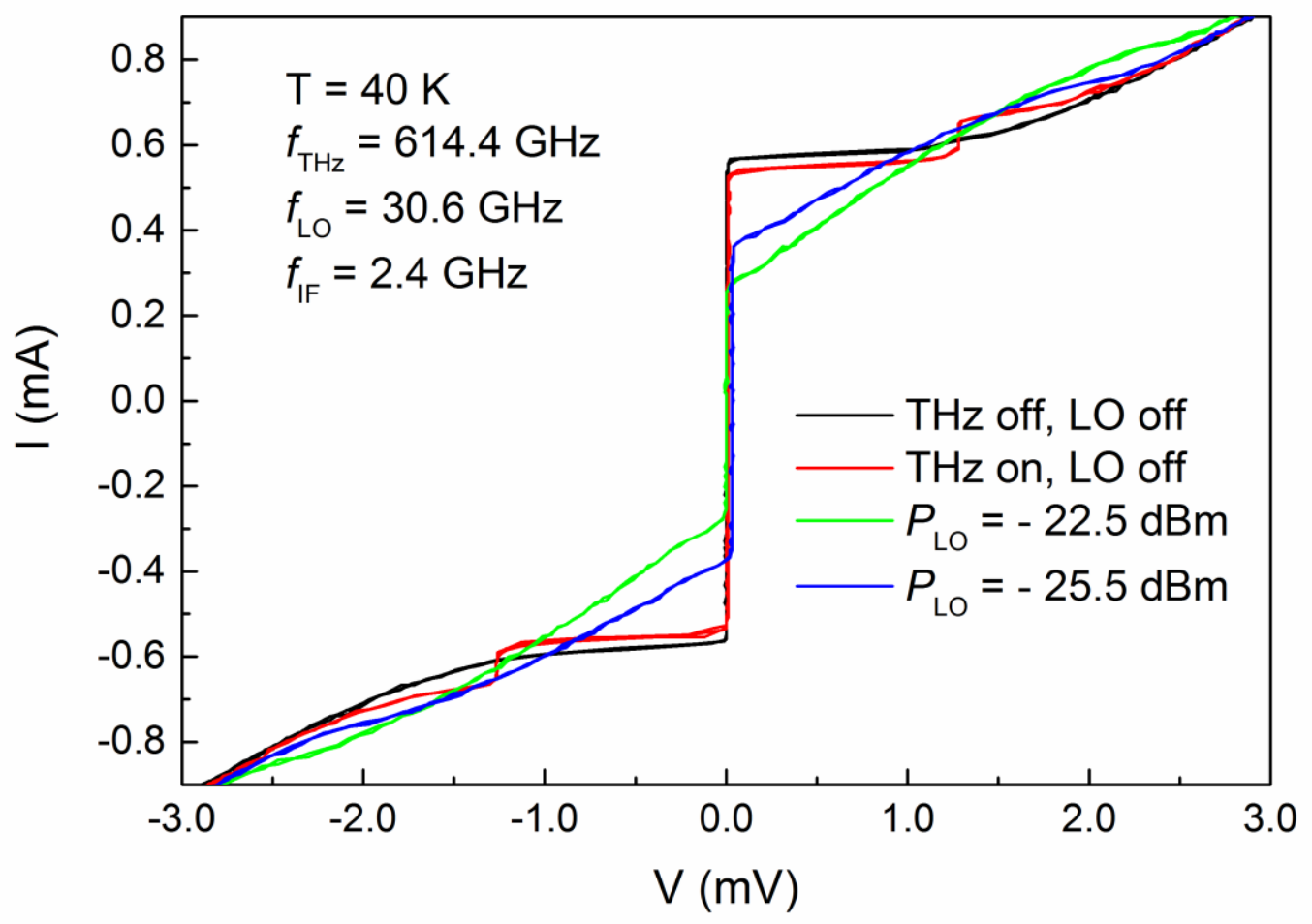

Figure 9. DC IVCS under the THz illumination and LO pumping, corresponding to the applied THz and LO (fundamental frequency) signal power for mixing results presented in figures 11 and 12.

\section{$\underline{\text { RF Measurement Results }}$}

Mixing properties have been studied for a range of $\mathrm{LO}$ and $\mathrm{THz}$ signal conditions at temperatures ranging from $40 \mathrm{~K}$ to $77 \mathrm{~K}$. For clarity, only a few selected results, mainly at $\mathrm{T}=40 \mathrm{~K}$, are presented in this paper. For harmonic mixer, $f_{\mathrm{IF}}=f_{\mathrm{THz}} \pm \mathrm{n} f_{\mathrm{LO}}$ (where $\mathrm{n}$ is the harmonic number). We have used two different LO frequencies in this experiment to mix with a THz signal of $f_{\mathrm{THz}}=614.4 \mathrm{GHz}$ : (1) $f_{\mathrm{LO}}=19.75$ $\mathrm{GHz}$, for the 31st order harmonic mixing product, $f_{\mathrm{IF} 1}=614.4-31 \times 19.75=2.15 \mathrm{GHz} ;(2) f_{\mathrm{LO}}=30.6$ $\mathrm{GHz}$, for the $20^{\text {th }}$ order harmonic mixing product, $f_{\mathrm{IF} 2}=614.4-20 \times 30.6=2.4 \mathrm{GHz}$. A higher IF output 
was obtained for the $20^{\text {th }}$ harmonic mixing. Figure 10 shows an example of a down-converted IF frequency spectrum at $2.4 \mathrm{GHz}$ for the $20^{\text {th }}$ order of harmonic mixing.

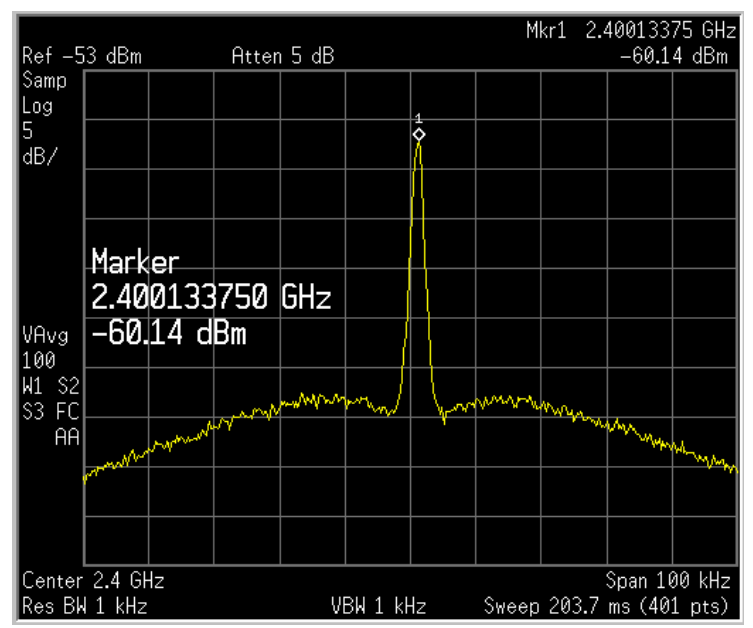

Figure 10. An IF frequency spectrum at $2.4 \mathrm{GHz}$ from the $20^{\text {th }}$ order harmonic mixing of a $30.6 \mathrm{GHz}$ LO signal with a THz signal of $614.4 \mathrm{GHz}$.

The operating conditions in terms of the DC current bias $I_{B}$ and LO power $P_{\mathrm{LO}}$ were studied. The IF output versus $I_{\mathrm{B}}$ and $P_{\mathrm{LO}}$ at $\mathrm{T}=40 \mathrm{~K}$ using $f_{\mathrm{LO}}=30.6 \mathrm{GHz}$ with $20^{\text {th }}$ order harmonic mixing are plotted in Figures 11 and 12, respectively. Figure 11 shows the mixer IF output power $P_{\text {IF }}$ as a function of the bias current $I_{B}$. The mixer works at a very wide range of current bias conditions and displays strong modulations whose periodicity is related to the quantized voltage between Shapiro steps induced by the $f_{\text {Lo. }}$ This will be further studied and simulated in the on-going work. Figure 11 also shows that both the IF output power and operating biasing range increase with increasing LO power. This can be qualitatively seen from the DC IVCs shown in Figure 9, where a higher LO power results in higher $I_{c}$ suppression by $P_{\mathrm{LO}}$ and therefore a wider $I_{\mathrm{B}}$ operating range. Figure 12 shows the $20^{\text {th }}$ mixing IF output against LO power (at fundamental frequency). It increases with increasing LO power and intends to plantae (or slow increase) at higher LO power. It shows a maximum peak at a LO power of - $22.5 \mathrm{dBm}$ and $P_{\mathrm{LO}} \geq-23 \mathrm{dBm}$ is required for optimal IF output or conversion efficiency. 


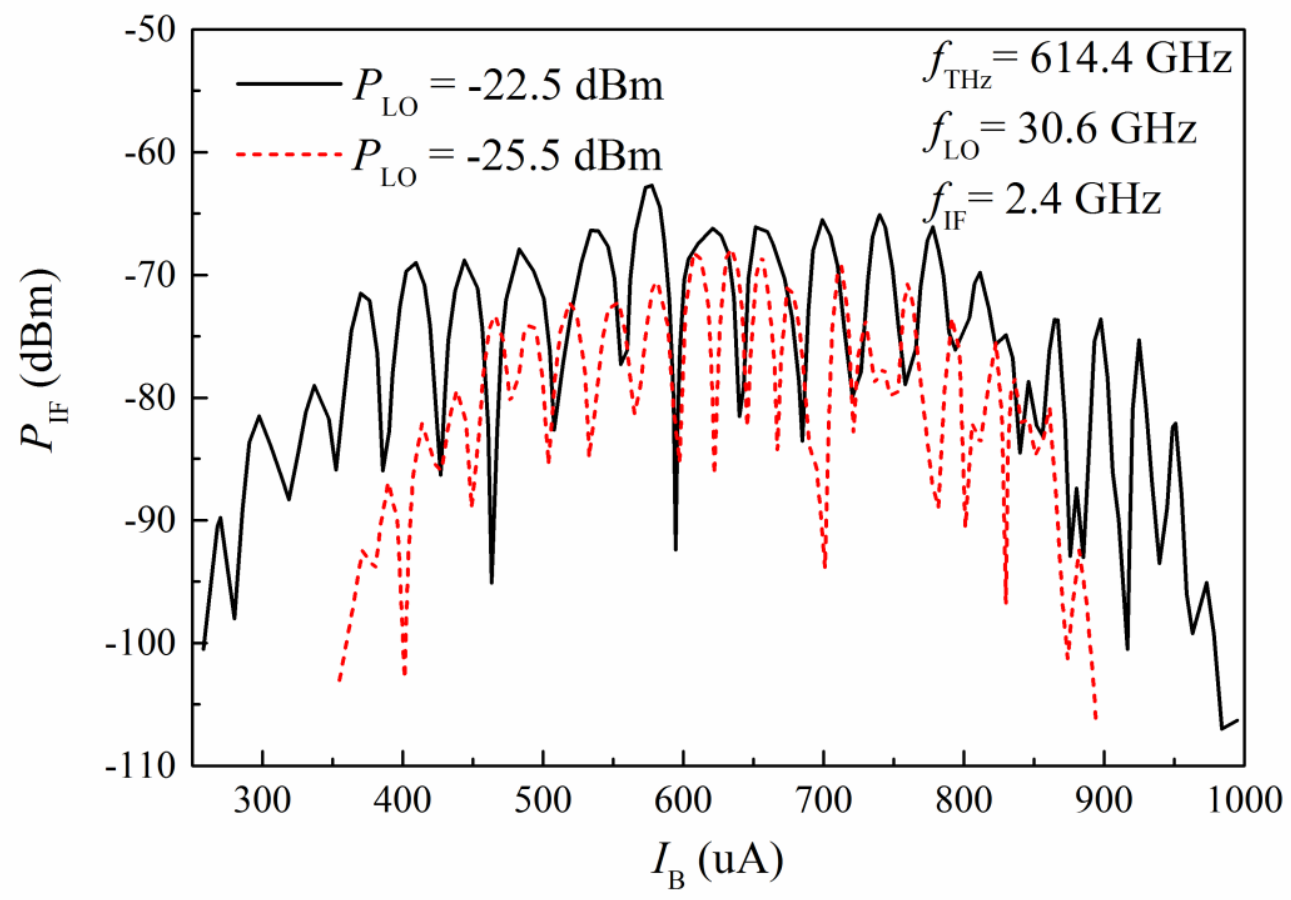

Figure 11: The $20^{\text {th }}$ order harmonic mixing IF output versus the bias current at $40 \mathrm{~K}$ for two different LO power levels.

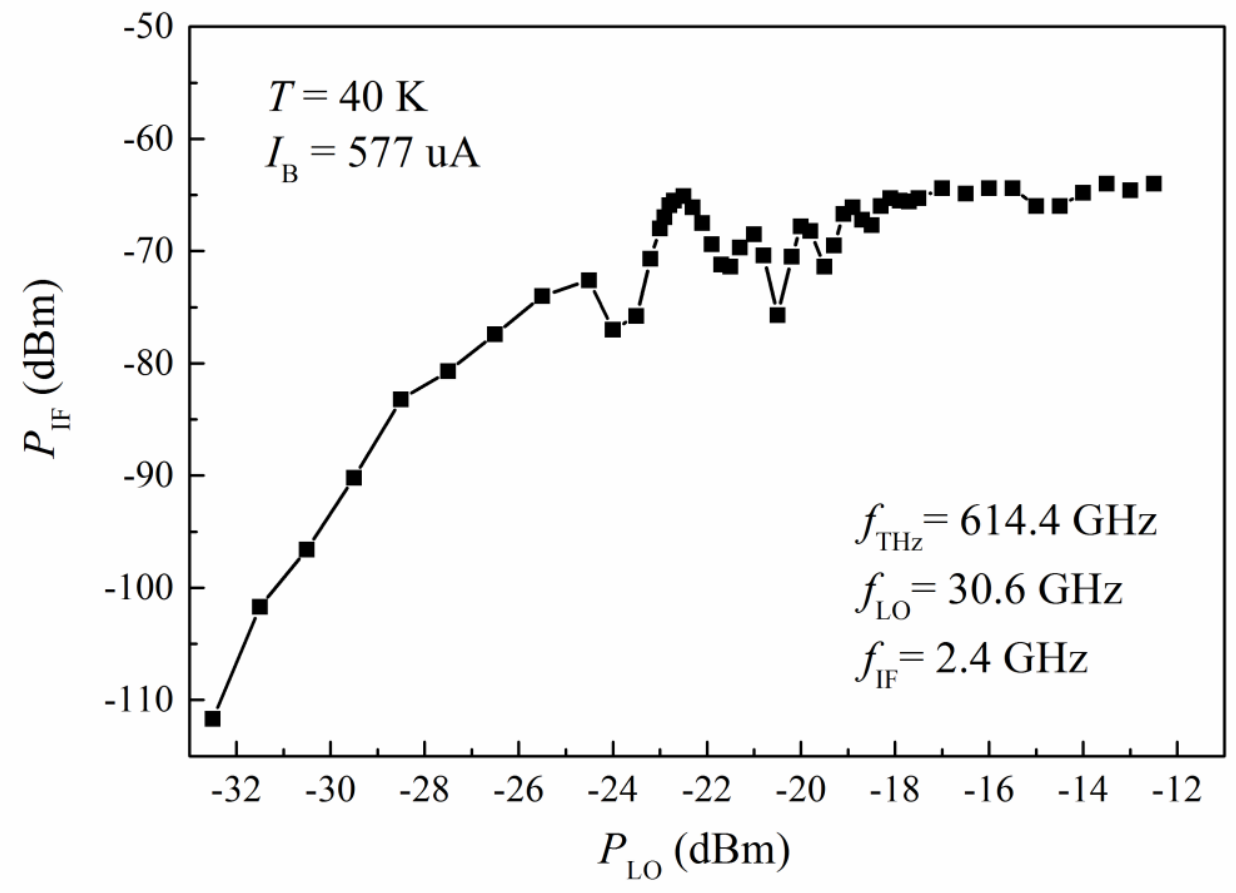

Figure 12. The $20^{\text {th }}$ order harmonic mixing IF output versus LO power (at fundamental frequency) at $40 \mathrm{~K}$ and at fixed bias current $I_{B}$. 
Using the estimated THz power ( $47 \mathrm{nW}$ ) coupled into the mixer junction from Fig. 9, a conversion efficiency of approximately $-49 \mathrm{~dB}$ is obtained for the $20^{\text {th }}$ order harmonic mixing (at $P_{\mathrm{LO}}=-22.5 \mathrm{dBm}$ and $I_{B}=577 \mu \mathrm{A}$ ). This value includes the junction IF output coupling loss and the loss of IF matching circuit chain (between ports P1 and P1 in Figure 2). This value is close to that of the state-of-art semiconductor Schottky diode harmonic mixers developed by Virginia Diode, Inc. ( - 45 dB at 500 GHz) [18] (no date available for $>600 \mathrm{GHz}$ high-order harmonic mixer). Further study of the mixer conversion efficiency versus different harmonic numbers is currently underway.

The presented mixer demonstrated stable operation at temperatures up to $77 \mathrm{~K}$, even for 31st order harmonic mixing. Figure 13 shows IF output spectra for the mixer operating at 60,70 and $77 \mathrm{~K}$. The IF output or conversion gain increases with decreasing temperature due to increasing dynamic resistance and junction characteristic voltage or frequency. Nevertheless, the mixer was able to operate at 77K reliably, indicating the potential of operating the HTS THz mixer in liquid nitrogen or a miniature single-stage cryocooler. In contrast, many reported HTS Josephson millimeter or $\mathrm{THz}$ mixers including those based on fundamental LO frequency mixing operated at temperatures well below 77K. This result shows the excellent quality of our step-edge junction coupled with a welldesigned antenna and matching/isolation circuit, which maximizes the mixer conversion efficiency.

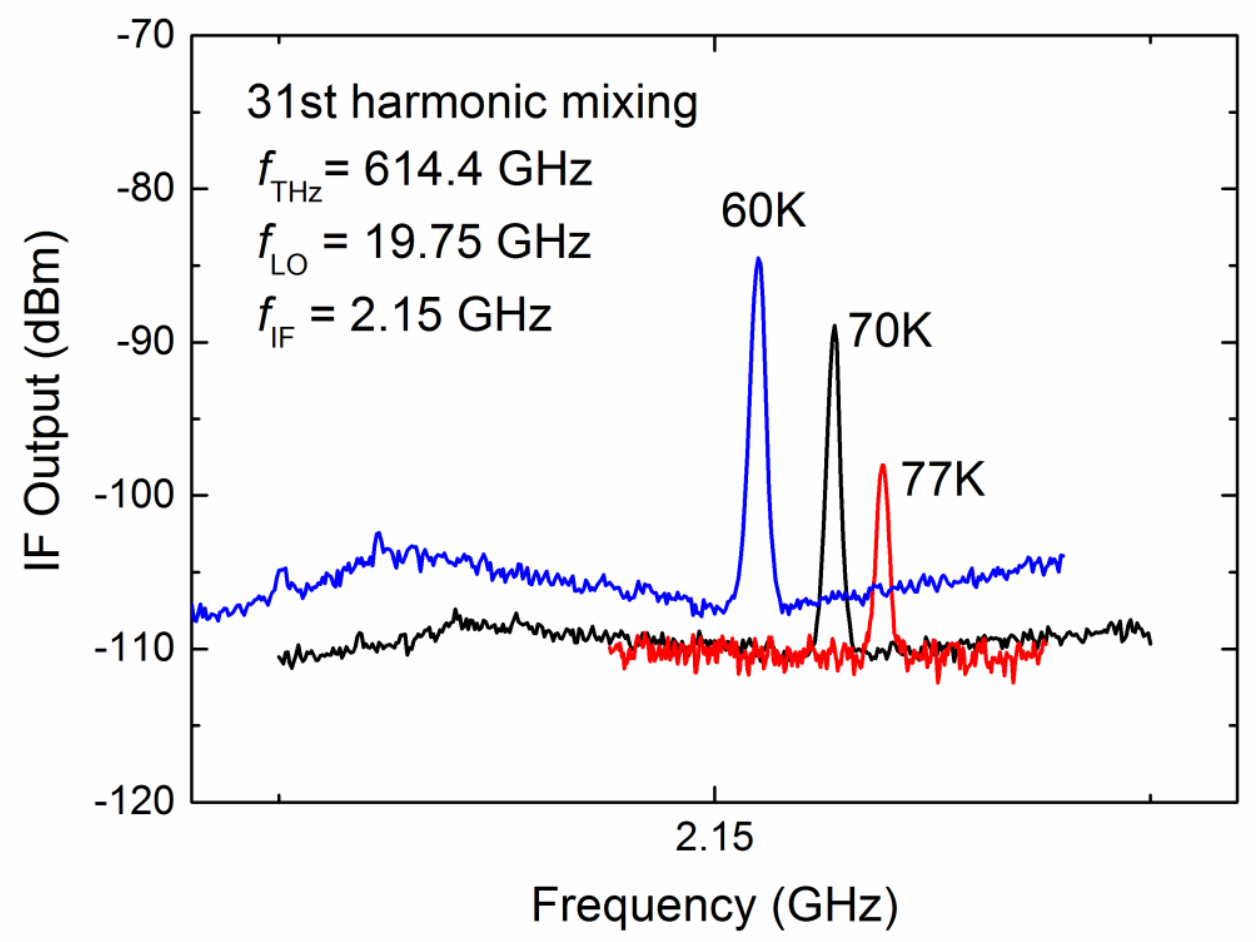

Figure 13. The $31^{\text {th }}$ harmonic mixing IF output spectrum at 60,70 and $77 \mathrm{~K}$. Note that the peaks are intentionally shifted slightly on the $x$-axis (frequency) for clarity. 
The linearity of the harmonic mixer was also measured at low $\mathrm{THz}$ power range and the result is shown in Figure 14. Note that this measurement was carried out on a different step-edge junction mixer which has a lower $I_{\mathrm{c}}$ value so a lower $P_{\mathrm{LO}}$ is required for optimal biasing. The THz power was largely attenuated from the maximum signal level by using a wire grid (and cardboard attenuators. A linear relationship is displayed at lower signal power range.

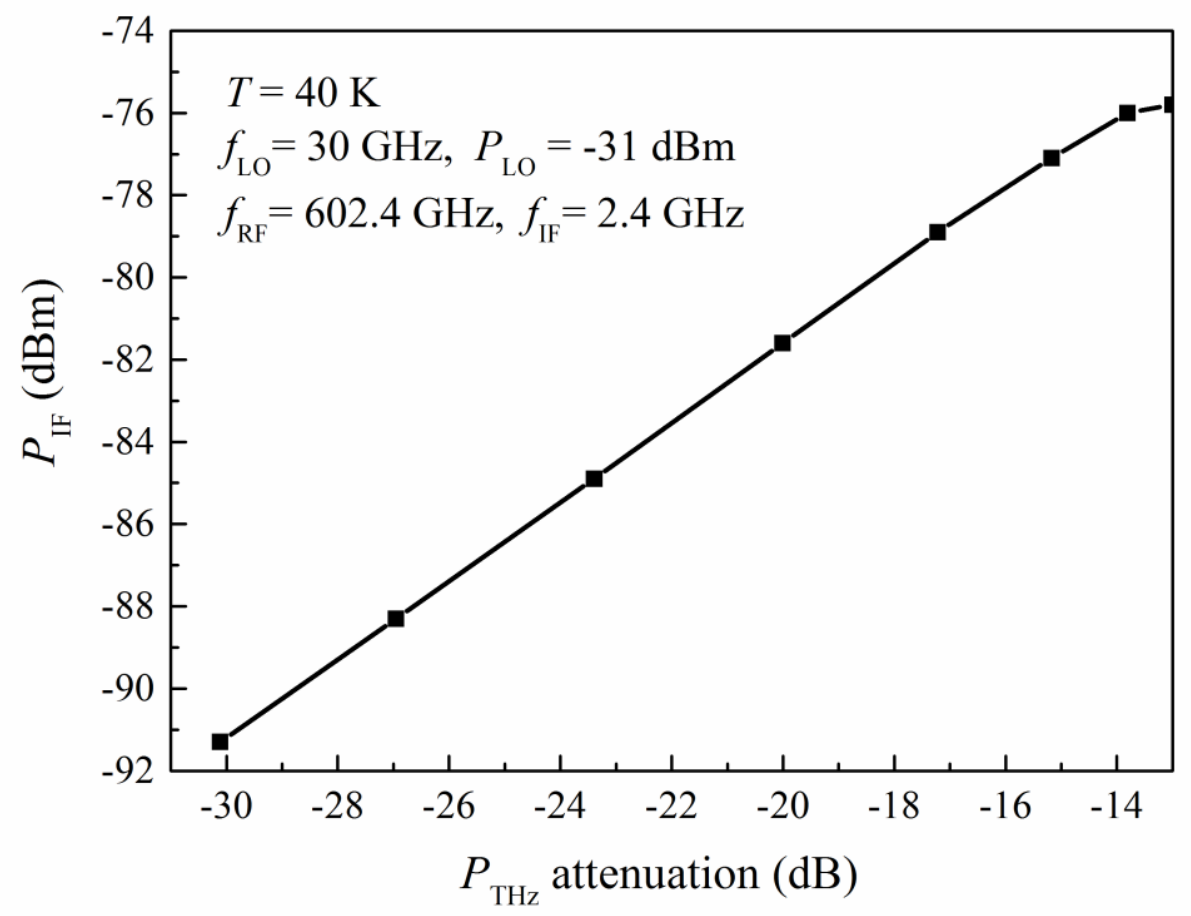

Figure 14. IF output versus the input $\mathrm{THz}$ power change (attenuated using different wire grid angle plus another power attenuator).

\section{Conclusion}

A thin-film antenna coupled YBCO step-edge Josephson junction heterodyne $\mathrm{THz}$ mixer was designed and experimentally demonstrated. The frequency down-conversion from $600 \mathrm{GHz}$ to $\sim 2 \mathrm{GHz}$ was obtained using high-order (up to 31st) harmonic mixing. The measured junction parameters showed excellent characteristics with its characteristic frequency reaches $\sim 1 \mathrm{THz}$ at $40 \mathrm{~K}$, thus suitable for $\mathrm{THz}$ frequency down-conversion. The mixer works under a wide range of bias current and LO power conditions and displays a linearity of the IF output power versus the $\mathrm{THz}$ signal power at low signal power range. The desirable junction characteristics coupled with a well-designed antenna and matching/isolation circuit enable successful operation of the mixer at relatively high temperatures 
(up to 77K) and maximize the conversion efficiency. The results demonstrated the potential of the HTS Josephson mixer for $\mathrm{THz}$ detection and $\mathrm{THz}$ communication receivers.

\section{Acknowledgement}

We thank Dr Stephen Hanham of Imperial College (U.K.) for advice on antenna design, Ms Jeina Lazar (CSIRO) for HTS chip fabrication and Ms Mei Shen (CSIRO) for packaging the module.

\section{References}

1. Kleine-Ostmann T and Nagatsuma T 2011 A Review on Terahertz Communications Research, J Infrared Milli Terahz Waves 32 143-171.

2. Chen J, Kurigata Y, Wang H B, Nakajima K, Yamashita T, and Wu P H 2003 Wideband frequency metrology using high temperature superconducting Josephson junctions, IEEE Trans. Appl. Supercond. 13, 1143-1146.

3. Divin Y Y, Poppe U, Volkov O Y, and Pavlovskii V V 2000 Frequency-selective incoherent detection of terahertz radiation by high-Tc Josephson junctions Appl. Phys. Lett. 76, 28262828 .

4. Du J, Smart K, Li L, Leslie K E, Wang D, Foley C P, Ji F, Li X D and Zeng D Z 2015 A cryogen-free HTS Josephson junction detector for terahertz imaging, Supercond. Sci. Technol. 28084001.

5. Du J, Hellicar A D, Li L, Hanham S, Macfarlane J C, Leslie K E, Nikolic N, Foley C P, and Greene K J 2009 Terahertz imaging at 77 K Supercond. Sci. Technol. 22114001.

6. Zhang T, Du J, Wang J, Bai D D, Guo Y J and He Y S 201530 GHz HTS Receiver Front-end based on Monolithic Josephson Mixer IEEE Trans Appl. Supercond. 251400605.

7. Du J, Zhang T, Guo Y J and Sun X W 2013 A high-temperature superconducting monolithic microwave integrated Josephson down-converter with high conversion efficiency, Appl. Phys. Lett. 102212602.

8. Harnack O, Darula M, Scherbel J, Heinsohn J K, Siegel M, DiehI D and Zimmermann P 1999 Optimization of a $115 \mathrm{GHz}$ waveguide mixer based on an HTS Josephson junction Supercond. Sci. Technol. 12 847-849. 
9. Scherbel J, Darula M, Harnack O and Siegel M 2002 Noise properties of HTS Josephson mixers at $345 \mathrm{GHz}$ and operating temperatures at $20 \mathrm{~K} \mathrm{IEEE} \mathrm{Trans.} \mathrm{Appl.} \mathrm{Supercon.} 12$ 18281831.

10. Malnou M, Feuillet-Palma C, Ulysse C, Faini G, Febvre P, Sorena M, Olanier L, Lesueur J and Bergeal N 2014 High-Tc superconducting Josephson mixers for terahertz heterodyne detection J. Appl. Phys. 116074505.

11. Taur Y, Claassen J H and Richards P L 1974 Conversion gain and noise in a Josephson mixer Rev. Phys. Appl. 9263.

12. Kita S and Fujisawa K 1982 Performance of Josephson junction harmonic mixers with harmonic number 1-8 at 70GHz Jap. J. Appl Phys. 21 497-503.

13. Chen J, Kobayashi E, Nakajima K, Yamashita T, Linzen S, Schmidl F and Seidel P 1999 Terahertz responses of HTS Josephson junctions on bicrystal substrates Advances in Superconductivity XI, eds. N. Koshizuka et al, Springer Japan, 1999, pp.1279-1284.

14. Foley C P, Mitchell E E, Lam S K H, Sankrithyan B, Wilson Y M, Tilbrook D L, and Morris S J 1999 Fabrication and characterisation of YBCO single grain boundary step edge junctions IEEE Trans. Appl. Supercond. 9 4281-4284.

15. Mitchell E E and Foley C P 2010 YBCO Step-edge junctions with high IcRn Supercond. Sci. Technol 23065007.

16. Du J, Lam S K H, and Tilbrook D L 2001 Metallization and interconnection of HTS YBCO thin film devices and circuits, Supercon. Sci. \& Technol 14 820-825.

17. Van Duzer T and Turner C W 1999 Superconductive Devices and Circuits, Prentice-Hall, Inc.

18. "Even Harmonic Mixers", Virginia Diodes, Inc. http://www.vadiodes.com/en/products/mixers 